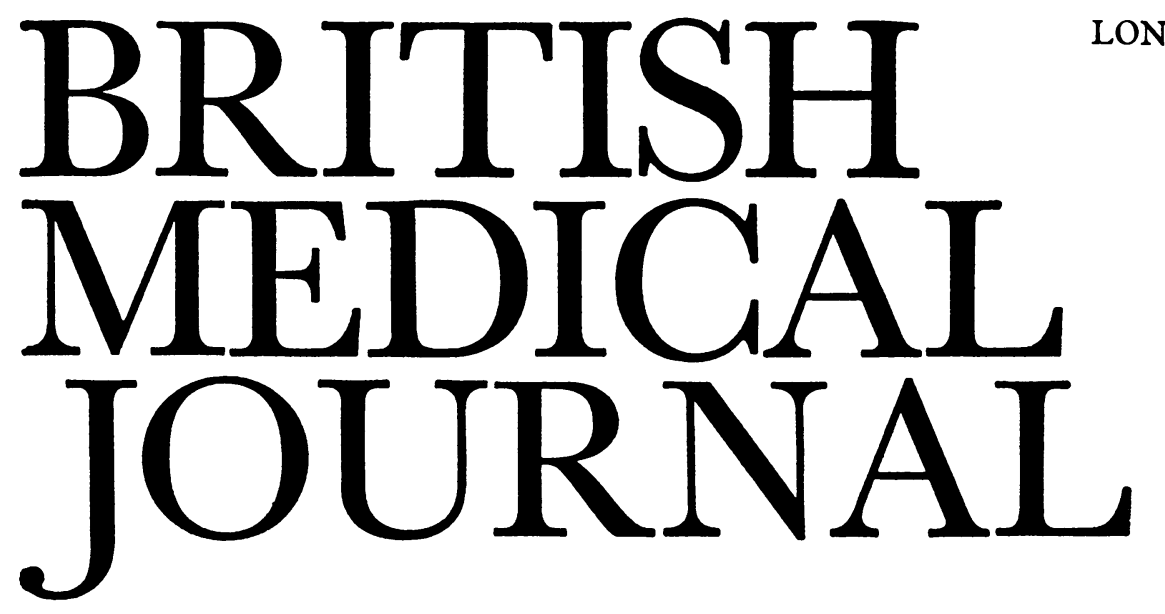

\title{
Venous thromboembolism and anticoagulants in pregnancy
}

Pulmonary embolism remains the second most common cause of maternal mortality and accounts for 30 deaths in every million deliveries. ${ }^{1}$ Two-thirds of these deaths occur in the puerperium, and the risk of puerperal venous thromboembolism is known to be enhanced by caesarean section, assisted delivery, the suppression of lactation by oestrogens, and advanced maternal age. ${ }^{12}$ Of these risk factors caesarean section is particularly important-the frequency of fatal pulmonary embolism is about nine times greater after caesarean than after vaginal delivery. Fortunately the management of venous thromboembolism during the puerperium is relatively straightforward and deaths from this cause have fallen considerably in recent years. Patients judged to be at high risk may now be monitored for venous thrombosis by the ${ }^{125}$ I-fibrinogen uptake test (if they are not breastfeeding their babies), and there is no contraindication to confirming or refuting the presence of clinically suspected venous thrombosis by venography. Anticoagulant treatment presents no serious problems, though there may be haemorrhagic complications such as bleeding from episiotomy wounds.

The reduction in deaths from puerperal pulmonary embolism has not been paralleled by a reduction during pregnancyprobably because the presence of the fetus imposes limitations on both diagnosis and treatment. For example, venography is precluded by the radiation risks to the fetus, and the ${ }^{125} \mathrm{I}-$ fibrinogen uptake test cannot be used because free isotopic iodine crosses the placenta and concentrates in the fetal thyroid. ${ }^{3}$ So the diagnosis of venous thrombosis has to be based on clinical signs, which are notoriously unreliablefor discomfort in the legs and ankle swelling often occur during pregnancy for reasons other than venous thrombosis. Pulmonary embolism may be the first indication of the presence of venous thrombosis, but minor chest symptoms may be attributed to pleurisy and a chest radiograph may be normal or fail to differentiate between infection and infarction. As a result the obstetrician often has to make a decision about treatment in the absence of a clear-cut diagnosis. Anticoagulants form the basis of treatment, though their use in pregnancy has not yet been justified by controlled clinical studies but only by the retrospective analysis of accumulated case reports.

The most extensive review before 1965 was undertaken by Villasanta, ${ }^{4}$ who found that maternal mortality from clinically diagnosed antepartum venous thromboembolism not treated with anticoagulants was about $13 \%$, whereas in patients treated with anticoagulants it was less than $1 \%$. Bleeding complications in treated mothers were not a serious problem, but there was a perinatal mortality of $18 \%$ in their offspring. The use of coumarin compounds (which cross the placenta) was the only constant factor associated with perinatal deathheparin seemed to be relatively safe. A more detailed analysis of the data implicating coumarins as a cause of perinatal mortality taken together with their own experimental and clinical observations led Hirsh et al ${ }^{5}$ to conclude that the problem resulted mainly from fetal haemorrhage induced by birth trauma in cases when the mother had received a coumarin compound until term. They suggested that such agents might safely be used in pregnancy if heparin was substituted three weeks before term.

The value of substituting heparin for warfarin in late gestation in preventing neonatal bleeding complications and perinatal death has been confirmed recently by Ramsey. ${ }^{6}$ A regimen for managing venous thromboembolism could therefore be: treat the initial episode with heparin as in the non-pregnant state; use warfarin up to the 37th week of gestation and then substitute heparin; stop treatment with heparin about six hours before delivery and begin it again six hours after delivery; and, finally, reintroduce warfarin two to seven days later. How long prophylaxis should be continued is not clear, but it is common practice to continue treatment for at least six weeks.

This pattern of treatment combines the convenience of oral anticoagulants with minimal risks of bleeding, and it would be the treatment of choice were it not that recent reports have implicated coumarin compounds as a cause of congenital malformations. ${ }^{7-11}$ These malformations include an unusual form of chondrodysplasia, epiphysial stippling, optic atrophy, and mental retardation; in each of the seven reported cases the mother had received an oral anticoagulant (warfarin in six, phenindione in one) either during the first trimester or throughout pregnancy. In every case the indication for treatment had been prophylaxis against systemic embolism in patients with rheumatic heart disease, four of the seven having had valve prosthesis implantations.

At present the evidence that coumarin compounds are teratogenic may be regarded as strong but not incontrovertible -in each of the seven cases there were factors other than oral anticoagulant therapy which have to be considered as potential causes of fetal malformation. Nevertheless, it seems prudent to use heparin and to avoid oral anticoagulants for thromboembolic problems during the first trimester, when the risk of teratogenic effects is greatest. Whether to use a coumarin compound after the first trimester is an open question, and opinions are likely to differ, but no cases of fetal malforma- 
tion have been reported in patients treated by the heparin/ warfarin regimen. ${ }^{56}$

If treatment with oral anticoagulants is to be avoided completely and if anticoagulant prophylaxis during the remainder of pregnancy and in the puerperium is necessary the only alternative is heparin. No teratogenic effects have been ascribed to heparin, and indeed these would not be expected from a drug which does not enter the fetal circulation. Unfortunately the need for parenteral administration is a considerable disadvantage, particularly if venous thromboembolism occurs early in pregnancy and the duration of treatment has to be prolonged, though women may be managed as outpatients if they are taught the technique of subcutaneous self-administration. The optimum dose of heparin for prophylaxis during pregnancy is not known, but 5000 units every eight hours produces peak heparin levels varying between 0.02 and 0.8 units $\mathrm{ml}$ in obstetric patients. Bleeding complications have been seen in patients with heparin levels above 0.6 units $\mathrm{ml}$, and Bonnar and Denson recommend regular monitoring of heparin concentrations. These workers have described a method for this which is not influenced by the raised levels of clotting factors which normally occur in pregnancy..$^{12} 13$

\footnotetext{
1 Department of Health and Locial Security, Confidential Enquiries into Maternal Deaths in England and Wales 1967-69. London, HMSO, 1972. Daniel, D G, Campbell, H, and Turnbull, A C, Lancet, 1967, 2, 287.

${ }^{3}$ Friend, J R, and Kakkar, V V, in Thromboembolism: Diagnosis and Treatment, ed V V Kakkar and A J Jouhar, p 131. Edinburgh and London, Churchill Livingstone, 1972.

4 Villasanta, U, American fournal of Obstetrics and Gynecology, 1965, 93, 142.

${ }^{5}$ Hirsh, J, Cade, J F, and O'Sullivan, E F, British Medical fournal, 1970, 1, 270.

${ }^{6}$ Ramsey, D M, Obstetrics and Gynecology, 1975, 45, 129.

7 DiSaia, P J, Obstetrics and Gynecology, 1966, 28, 469.

${ }^{8}$ Kerber, I J, Warr, O S, and Richardson, C, fournal of the American Medical Association, 1968, 203, 223.

${ }^{9}$ Becker, M H, et al, American fournal of Diseases of Children, 1975, 129, 356.

10 Shaul, W L, Emery, H, and Hall, J G, American fournal of Diseases of Children, 1975, 129, 360.

11 Pettifor, J M, and Benson, R, Fournal of Pediatrics, 1975, 86, 459.

12 Bonnar, J, and Denson, K W E, Lancet, 1974, 2, 956.

${ }^{13}$ Denson, K W E, and Bonnar, J, Thrombosis et Diathesis Haemorrhagica 1973, 30, 471 .
}

\section{Prognosis and treatment in Perthes's disease}

Sound advice on the treatment of Perthes's disease is still difficult to give. In childhood the symptoms are mild and transient and subside quickly with bed rest. Treatment is inspired mainly by the fear that deformation of the femoral head will lead to osteoarthritis in adult life. The little information we have on the extent of this risk has not been alarming: follow-up 30 years after diagnosis showed few patients with any symptoms, though less than half had hips that were radiologically normal. ${ }^{2}$ If or when osteoarthritis supervenes surgery already has much to offer, and by the time our present patients with Perthes's disease need help treatment may be better still. The management of the disease in childhood has to be viewed against this background.

Treatment aimed at preserving the shape of the femoral head is apt to be strenuous and may mean two or more years of invalidism. Furthermore, it has been by no means always successful, while without any treatment some children have recovered a good round femoral head. The outlook for those who present late with a deformed cartilaginous head is poor and unlikely to be altered by the management given. For the rest Catterall's studies ${ }^{3}$ (developing an observation of $\mathrm{O}^{\prime}$ Garra ${ }^{4}$ ) showed that the prognosis was much influenced by the extent of the original infarction or infarctions. When only the front part of the epiphysis was affected little deformation resulted even without treatment. When all or nearly all the epiphysis was affected nearly half had poor results. Unfortunately for science the number of Catterall's patients in the severe grades was too small to test the value of treatment convincingly. A larger series has now been reported," and the usefulness of Catterall's criteria has been confirmed. The results of treatment by containment-by placing the affected epiphysis entirely in the mould of the acetabular cup by abduction and internal rotation of the hip-were compared with the results of non-containment treatment such as traction in bed or the use of a weight-relieving calliper or sling. Of 60 patients in the severer grades and not treated by containment 33 ended with a femoral head that was not round, while of 36 similar patients treated by containment only 10 had a poor result. Of the less severely affected hips 19 treated by containment did little better than 57 treated by other methods.

It seems, therefore, that when only the front part of the epiphysis is affected the type of treatment is probably unimportant, and perhaps none at all is really needed. There will sometimes be difficulty, particularly in an early case, in judging the extent of epiphysial damage. Until this is clear the epiphysis has to be protected, for with more extensive disease the evidence shows that treatment helps. In theory, in experiment, ${ }^{6}$ and in practice containment methods offer the greatest advantage: in one study $^{7}$ changing from simple protection from weight-bearing to the use of abduction plasters reduced the incidence of poor results from $35^{\circ} \%$ to $9^{\circ}$, though the comparability of the later patients with the earlier was not established with the detail that now seems desirable. The children were not confined to bed by their abduction plasters, but the treatment was very disabling and lasted an average of 19 months. Such prolonged invalidism may be avoided if containment is achieved by subtrochanteric osteotomy designed to place the femoral head epiphysis entirely in the acetabulum when standing. " This surgically induced coxa vara shortens the leg a little and itself causes a limp, but this corrects itself later with growth. Once the osteotomy is united there is no need to restrict the child's activity. The same effect may be obtained with slightly more difficulty by pelvic osteotomy," tilting the acetabulum to cover the femoral head fully. Applied to severe cases femoral osteotomy produced five good results in nine patients ${ }^{10}$ and pelvic osteotomy 11 satisfactory out of $15,{ }^{9}$ results comparable with those from other containment techniques.

Until we are able to control the original pathological defect in Perthes's disease, the cause of the damage to the femoral head, our methods of preventing deformation will remain relatively crude. We can now recognise that considerable proportion of the patients who have an inherently good prognosis and for whom strenuous treatment appears not to be indicated. For the others our therapeutic zeal should be tempered by recognition of the deficiencies of our current techniques, especially now that operations for hip replacement have reduced the seriousness of hip arthritis in adults.

${ }^{1}$ Ratliff, A H C, fournal of Bone and foint Surgery, 1967, 49B, 102.

2 Danielsson, L G, and Hernborg, J, Acta Orthopaedica Scandinavica, $1965,36,70$.

3 Catterall, A, Fournal of Bone and foint Surgery', 1971, 53B, 37.

+ O'Garra, J A, Fournal of Bone and foint Surgery, 1959, 41B. 465. 